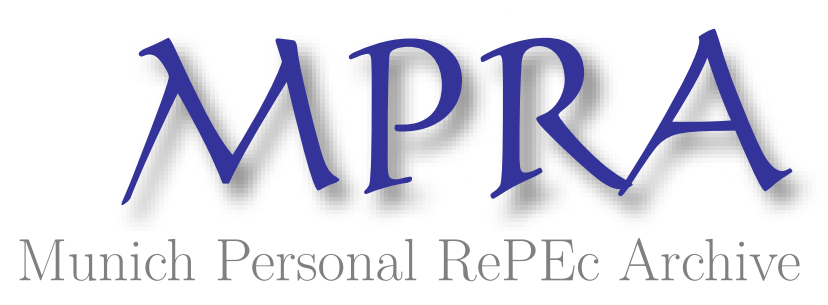

Growth and Keeping up with the Joneses

Wendner, Ronald

Graz University, Austria

19 October 2009

Online at https://mpra.ub.uni-muenchen.de/18001/

MPRA Paper No. 18001, posted 22 Oct 2009 09:54 UTC 


\title{
Growth and Keeping up with the Joneses
}

\author{
Ronald Wendner *
}

Department of Economics, University of Graz, Austria

Universitaetsstrasse 15, RESOWI-F4,

A-8010 Graz, Austria

E-mail ronald.wendner@uni-graz.at

Web http://www.uni-graz.at/ronald.wendner

Phone +433163803458

October 19, 2009

\begin{abstract}
This paper investigates the impact of the desire to keep up with the Joneses (KUJ) on economic growth and optimal tax policy in a continuous time overlapping generations model with AK technology and gradual retirement. Due to the desire to KUJ, the propensity to consume out of total wealth rises (declines), and the balanced growth rate declines (increases) when the households' individual total (accumulated and human) wealths are increasing (decreasing) with age. The rate of retirement determines whether or not a household's total wealth is increasing with age. If total wealth is increasing (decreasing) with age, an optimal allocation is decentralized by an intergenerationally progressive (regressive) lump sum tax system. The desire to KUJ strengthens the intergenerational regressivity (progressivity) of the optimal tax system.
\end{abstract}

Keywords and Phrases: Keeping up with the Joneses, AK growth, overlapping generations, gradual retirement, optimal taxation.

JEL Classification Numbers: D91, E21, O40

\footnotetext{
${ }^{\star}$ I thank Robert Hill, Christian Groth, and Karl Farmer for significant comments on a previous draft. I retain sole responsibility for any remaining errors.
} 


\section{Introduction}

Psychologists have often pointed to the fact that individuals experience happiness by doing well relative to some reference group. In economic terms, this observation refers to the desire to keep up with the Joneses. That is, individual utility can depend directly on the current levels of consumption of others. This paper analyzes the theoretical impact of the desire to keep up with the Joneses on growth of consumption and output, and on optimal taxation (along a balanced growth path).

Preferences do exhibit the desire to keep up with the Joneses (KUJ). This is shown in several recent studies that aim to assess empirically the extent to which individual utility depends on others' consumptions. These studies are based on survey-experimental methods, and on econometric analyses of panel data on individuals' incomes and self reported happiness. ${ }^{1}$

The theoretical impact of the desire to KUJ on growth of consumption and output was analyzed in several papers. The results are, however, mixed. Brekke and Howarth (2002, p.142) and Rauscher (1997) find that augmenting a standard neoclassical representative agent model with the desire to KUJ has no effects on long-run behavior. In a framework where individual utility depends on a consumption reference stock rather than on the current levels of consumption of others ("catching up with the Joneses"), Alvarez-Cuadrado et al. (2004) show that the desire to catch up with the Joneses raises (lowers) the consumption growth rate if consumption exceeds (is lower than) the consumption reference stock. In the presence of capital externalities (endogenous growth) the desire to KUJ results in an increase in the balanced growth rate (Brekke and Howarth 2002, Carroll et al. 1997, Corneo and Jeanne 1997, Liu and Turnovsky 2005). In the context of a continuous time overlapping generations model with neoclassical production technology (without a production externality), Fisher and Heijdra (2009) and Wendner (2009) show that the desire to KUJ can reduce individual steady state consumption growth rates. Initially, the desire to

\footnotetext{
${ }^{1}$ Alpizar et al. (2005), Carlsson et al. (2003), Ferrer-i-Carbonell (2005), Johansson-Stenman et al. (2002, 2006), Luttmer (2005), McBride (2001), Neumark and Postlewaite (1998), Solnick and Hemenway $(1998,2005)$.
} 
KUJ may induce households to raise their consumption levels. The intertemporal budget constraint, then, requires them to lower their steady state consumption growth rates.

This paper considers the impact of the desire to KUJ on consumption and output growth within the framework of a continuous time overlapping generations model with AK technology, gradual retirement, and exogenous labor supply. ${ }^{2}$ By setting the birth rate equal to zero, the framework yields the representative agent economy, as a special case. The model reproduces many of the results given in the prior literature, as indicated above. In addition, the model offers conditions for the desire to KUJ to raise (lower or not affect) the balanced growth rates. The implications for optimal tax policy are discussed in detail.

The key factor determining the impact of the desire to KUJ on the balanced growth rates of consumption and output is the development of total - that is, accumulated and human - individual wealth with age. Total individual wealth may decrease or increase with age, depending on the rate of gradual retirement. For a low rate of retirement, total wealth increases with age. But for a high (enough) rate of retirement, human wealth declines so strongly with age that also total wealth declines with age. Many of the results of this paper depend on whether or not the rate of retirement is "low" or "high enough" such that total wealth declines with age. A numerical estimate suggests that a "realistic" rate of retirement is possibly "high enough" (in the sense discussed above). Clearly, in a representative agent framework, wealth is independent of age. Thereby, the representative agent framework can be seen (under qualifications) as a knife-edge case of the continuous time overlapping generations framework developed below.

The paper shows two main results. First, at a balanced growth path, whenever total individual wealth is nonconstant with age, every household responds to the desire to KUJ by either raising its propensity to consume out of wealth (thereby lowering its consumption growth rate) or vice versa. In particular, if total wealth rises with age, a household will respond to (an increase of) the desire to KUJ by raising its propensity to consume out of wealth (thereby by lowering its consumption growth rate). The reverse is true for the case

\footnotetext{
${ }^{2}$ This paper does not consider the desire to catch up with the Joneses.
} 
in which total wealth is declining with age.

This finding is explained by the fact that the desire to KUJ raises the concavity of a household's utility function. Households respond by shifting consumption to periods of low consumption - that is, to the presence in case wealth rises with age, and to the future in case wealth declines with age.

Second, those findings are reflected in an optimal tax program. Key to this program is a lump sum transfer system that involves intergenerational redistribution. The redistribution goes from old to young when total individual wealth increases with age, and it goes from young to old when total individual wealth decreases with age. In both cases, the desire to KUJ raises the optimal levels of intergenerational redistribution, which becomes more intergenerationally progressive (regressive) when individual wealth increases (decreases) with age.

Section 2 of this paper presents the market economy's structure. Section 3 discusses the impact of the desire to KUJ on balanced (market economy's and optimal) growth rates and on an optimal tax program. Section 4 concludes the paper. The appendix contains a number of derivations and proofs that were distracting when placed in the main text.

\section{Model Setup}

Population. An individual born at time $v$ ("vintage") is uncertain about the length of his or her life. As in Blanchard (1985), the instantaneous probability of death (the death rate, d) of a cohort is age-independent and constant over time. Also, the ratio of the expected number of births per time unit to the size of the population - the birth rate $(b)$ - is assumed to be age-independent and constant over time.

At time $t$, the population size is $L(t)$. At each instant of time, a new cohort is born, the size of which is $b L(t)$ per time unit. Also, at time $t$, the mass of people who die is $d L(t)$. Accordingly, for a large population size, the rate of population growth is $n=b-d$. Population at some date $t_{1}$ is given by: $L\left(t_{1}\right)=L\left(t_{0}\right) e^{n\left(t_{1}-t_{0}\right)}$. Without loss of generality, $L(0)=1$, and $L(t)=e^{n t}$.

Let $L(v, t)$ be the size of a vintage- $v$ cohort at time $t$. Under this population struc- 
ture $L(v, t)=L(v, v) e^{-d(t-v)}=b L(v) e^{-d(t-v)}=b e^{n v} e^{-d(t-v)}=b e^{b v-d t}$. Similarly, the share of a vintage- $v$ cohort in total population at time $t$ is: $l(v, t) \equiv L(v, t) / L(t)=$ $\left[b e^{b v} e^{-d t}\right] / e^{(b-d) t}=b e^{-b(t-v)}$.

Production. There is a unit mass of competitive, identical firms. Firm $i$ produces a homogeneous output, $Y_{i}$, according to

$$
Y_{i}(t)=\bar{A} K_{i}(t)^{\alpha}\left[E(t) \hat{L}_{i}(t)\right]^{1-\alpha}, \quad 0<\alpha<1,
$$

where $K_{i}$ is capital, $\hat{L}_{i}$ are labor services employed by firm $i$, and $E$ - which is considered exogenously given by individual firms - represents a measure for aggregate labor productivity.

We consider gradual (exponential) retirement. Labor supply $\hat{L}(v, t)$ per time unit at date $t$ of a cohort born at date $v$ depends on age, $(t-v)$, according to:

$$
\hat{L}(v, t)=L(v, t) e^{-\lambda(t-v)}=b e^{b v-d t} e^{-\lambda(t-v)}=b L(t) e^{-(b+\lambda)(t-v)}, \quad 0 \leq \lambda,
$$

where we refer to $\lambda$ as the retirement parameter. Labor supply and population size are related as follows to each other:

$$
\begin{aligned}
& \hat{L}(t)=\int_{-\infty}^{t} \hat{L}(v, t) d v=b L(t) \int_{-\infty}^{t} e^{-(b+\lambda)(t-v)} d v=\beta L(t), \\
& \text { where } \beta \equiv \frac{b}{b+\lambda} \in(0,1] .
\end{aligned}
$$

For a given population size, earlier retirement implies lower aggregate labor supply.

Given $\hat{L}_{i}(t)=\beta L_{i}(t)$, we can reformulate $(1)$ as:

$$
Y_{i}(t)=\bar{A} K_{i}(t)^{\alpha}\left[E(t) \beta L_{i}(t)\right]^{1-\alpha}
$$

Define the aggregate capital stock $K(t)=\int_{0}^{1} K_{i}(t) d i$. Labor productivity is given by:

$$
E(t)=K(t) \hat{L}(t)^{\varepsilon-1}, \quad 0<\varepsilon \leq 1
$$

where $\varepsilon$ is called the scale effect parameter. ${ }^{3}$ If $\varepsilon>0$, a rise in the level of labor raises labor productivity. The lower bound on $\varepsilon$ ensures positivity of the social marginal product

\footnotetext{
${ }^{3}$ This is a special case of a more general specification, which was introduced by Groth and Smulders (2008).
} 
of labor. If $\varepsilon>0$, a balanced growth path exists only for $n=0$, which we assume as of here. Then, without loss of generality, $L(t)=L_{0}=1$.

Firms maximize profits and hire factors from households on competitive factor markets. In equilibrium, in a competitive framework:

$$
\begin{aligned}
r(t)+\delta & =\frac{\partial Y_{i}(t)}{\partial K_{i}(t)}=\alpha \bar{A} E(t)^{1-\alpha} K_{i}(t)^{-(1-\alpha)} \hat{L}_{i}(t)^{1-\alpha}=\alpha A, \\
w(v, t) & =\frac{\partial Y_{i}(t)}{\partial\left[E(t) \hat{L}_{i}(t)\right]} \frac{\partial\left[E(t) \hat{L}_{i}(t)\right]}{\partial L_{i}(v, t)} \\
& =(1-\alpha) \bar{A} K_{i}(t)^{\alpha}\left[\hat{L}_{i}(t) E(t)\right]^{-\alpha} E(t) e^{-\lambda(t-v)} \\
& =(1-\alpha) A \beta^{-1} k(t) e^{-\lambda(t-v)},
\end{aligned}
$$

where $r(t)$ is the rate of interest, $w(v, t)$ is the wage rate, and $\delta$ is the rate of depreciation of capital. It follows: $w(v, t)=w(t, t) e^{-\lambda(t-v)}$. At date $t$, the "average wage" is given by: $w(t)=\int_{-\infty}^{t} l(v, t) w(v, t) d v=(1-\alpha) A k(t)$, thus, $w(v, t)=\beta^{-1} w(t) e^{-\lambda(t-v)}$.

Aggregate production is given by:

$$
Y(t) \equiv \int_{0}^{1} Y_{i}(t) d i=\bar{A} \beta^{(1-\alpha) \varepsilon} K(t) L(t)^{(1-\alpha) \varepsilon}
$$

where we use the fact that, in equilibrium, with profit maximizing firms, $K_{i} / L_{i}=K / L$. Define per capita output and capital: $y(t) \equiv Y(t) / L(t)$, and $k(t) \equiv K(t) / L(t)$, and consider $L(t)=1$ :

$$
y(t)=A k(t), \quad \text { where } A \equiv \bar{A} \beta^{(1-\alpha) \varepsilon}
$$

According to the resource constraint, $\dot{K}(t)=Y(t)-C(t)-\delta K(t)$, where $C(t)$ is aggregate consumption at date $t$. Let per capita consumption be $c(t) \equiv C(t) / L(t)$. Then, capital evolves according to:

$$
\dot{k}(t)=y(t)-c(t)-\delta k(t)
$$

Households. Time- $t$ instantaneous utility of a vintage- $v$ household is a function $u($.$) of$ own consumption $c(v, t)$ and reference consumption $c(t)$. The first argument in $c(v, t)$ refers to the birth date ("vintage"), and the second argument refers to time. At time $t$, an individual household may not only care about its own consumption, but also about 
how own consumption compares to the average consumption level, $c(t)$, which serves as a consumption reference level (as discussed below).

In this paper, we consider the standard case of a constant relative risk aversion (CRRA) utility function. We specify the felicity function as:

$$
u(c(v, t), c(t))=\frac{\left[c(v, t)^{\frac{1}{1-\eta}} c(t)^{-\frac{\eta}{1-\eta}}\right]^{1-\sigma}-1}{1-\sigma}=\frac{\left[c(v, t)\left(\frac{c(v, t)}{c(t)}\right)^{\frac{\eta}{1-\eta}}\right]^{1-\sigma}-1}{1-\sigma},
$$

where $0 \leq \eta<1$ is called the reference parameter, which measures the importance of the consumption reference level. If $\eta=0$, utility depends only on own consumption, and the model reduces to the usual model with interpersonally separable utility. The desire to KUJ is introduced by a positive value for $\eta$. If $\eta>0$, utility is also derived from own consumption relative to the reference level (average consumption).

The specification of the desire to KUJ is chosen to allow for the interpretation of the reference parameter as marginal degree of positionality (Johansson-Stenman et al., 2002). ${ }^{4}$ The reference parameter represents the fraction of marginal utility of consumption stemming from a rise in $c(v, t) / c(t)$. If, say, $\eta=0.2$, then $20 \%$ of marginal utility of consumption stems from a rise in the ratio $c(v, t) / c(t)$, while the remaining $80 \%$ directly come from a rise in own consumption $c(v, t)$, holding constant $c(v, t) / c(t)$.

Parameter $\sigma$ governs the intertemporal elasticity of substitution. If $\eta=0$, the intertemporal elasticity of substitution is given by $\sigma^{-1}$. If, however, $\eta>0$, both parameters, $\sigma$ and $\eta$ determine the elasticity of substitution between consumption at any two points in time.

We observe the following two sign restrictions. First, $0 \leq \eta<1$ ensures positive marginal utility of consumption and quasiconcavity of the utility function. Second, $\sigma>1$, which is overwhelmingly suggested by the literature, ensures decreasing marginal utility of individual consumption and strict concavity of $u($.$) in c(v, t)$. In particular, these sign restrictions imply:

$$
\hat{\sigma} \equiv \frac{(\sigma-\eta)}{1-\eta} \geq \sigma>1
$$

\footnotetext{
${ }^{4}$ Johansson-Stenman et al. (2002) call $\eta$ the "marginal degree of positionality." Formally, define $r(v, t)=c(v, t) / c(t)$, and $\tilde{u}(c(v, t), r(v, t)) \equiv u(c(v, t), c(t))$. Then $\eta=$ $[\partial \tilde{u}(.) / \partial r(v, t) \partial r(v, t) / \partial c(v, t)] /[(\partial \tilde{u}(.) / \partial c(v, t))+(\partial \tilde{u}(.) / \partial r(v, t) \partial r(v, t) / \partial c(v, t))]$.
} 
where $\hat{\sigma}$ represents the (absolute of the) elasticity of marginal utility, which increases in the desire to KUJ. Notice that the chosen specification is consistent with Dupor and Liu's (2003) definition of KUJ: $\partial^{2} u() /.[\partial c(v, t) \partial c(t)]>0$.

At time $t$, expected remaining lifetime utility of a cohort born at date $v$ is:

$$
U(v, t)=\int_{t}^{\infty} u(c(v, s), c(s)) e^{-(\rho+d)(s-t)} d s,
$$

where $\rho$ is the household's pure rate of time preference. The possibility of death $(d>0)$ leads to a discount rate $(\rho+d)$ higher than the pure rate of time preference.

Consumption. Households do not have a bequest motive. They can buy fair life annuity contracts from life insurance companies, for which they pay or receive the annuity rate of interest $r^{a}$. The contracts are canceled upon death of an individual. Actuarial fairness requires $r^{a}=r+d$. The annuity interest factor is given by: $R^{a}\left(t_{0}, t_{1}\right) \equiv \int_{t_{0}}^{t_{1}}[r(s)+d] d s=$ $(r+d)\left(t_{1}-t_{0}\right)$.

Every household inelastically supplies labor services and chooses consumption at all $t \geq v$ such as to maximize expected lifetime utility (11) subject to its intertemporal budget constraint: ${ }^{5}$

$$
a(v, t)+h(v, t)-\int_{t}^{\infty} c(v, s) e^{-R^{a}(t, s)} d s=0,
$$

where $a(v, t)$ stands for time- $t$ assets (accumulated wealth) of a vintage- $v$ household, and human wealth $h(v, t) \equiv \int_{t}^{\infty} w(v, s) e^{-R^{a}(t, s)} d s$ is the integral of discounted present and future wage payments. In the market framework, a household does not consider the impact of its individual consumption on the consumption reference level.

Individual consumption levels are derived by applying Pontryagin's maximum principle, in the appendix. Define the propensity to consume out of total wealth:

$$
\Delta^{-1}(t) \equiv\left[\int_{t}^{\infty} e^{\int_{t}^{s} \frac{r-\rho}{\hat{\sigma}}+\frac{\hat{\sigma}-\sigma}{\hat{\sigma}} \frac{\dot{c}(\tau)}{c(\tau)} d \tau-R^{a}(t, s)} d s\right]^{-1} .
$$

Then:

$$
c(v, t)=\Delta^{-1}[a(v, t)+h(v, t)], \quad c(t, t)=\Delta^{-1} h(t, t)=\Delta^{-1} \beta^{-1} h(t),
$$

\footnotetext{
5 The transversality condition required to prevent households from oversaving is: $\lim _{s \rightarrow \infty} e^{-R^{a}(t, s)} a(v, s)=0$, or, equivalently, $\lim _{s \rightarrow \infty} \mu(s) e^{-(\rho+d) s} a(v, s)=0$, where $\mu$ is the shadow price of accumulated wealth. Budget constraint (12) follows from combining the flow budget constraint, $\dot{a}(v, t)=r^{a} a(v, t)+w(v, t)-c(v, t)$, with the transversality condition.
} 
where the second expression follows from the fact that there is no operative bequest motive: $a(t, t)=0$. Consumption levels are proportional to total (accumulated and human) wealth, with the age-independent factor of proportionality given by: $\Delta^{-1}(t)$, which can be interpreted as the propensity to consume out of total wealth. Notice that at any given point in time, consumption levels are not equal across cohorts.

Consumption growth rates are equal across cohorts. Individual consumption growth rates are given by:

$$
g_{c(v, .)} \equiv \frac{\dot{c}(v, t)}{c(v, t)}=\frac{[r-\rho]}{\hat{\sigma}}+\frac{\hat{\sigma}-\sigma}{\hat{\sigma}} \frac{\dot{c}(t)}{c(t)}
$$

Average accumulated wealth, $a(t)$, is given by $a(t) \equiv \int_{-\infty}^{t} l(v, t) a(v, t) d v$. In a closed economy (without adjustment costs), the following identity holds:

$$
a(t)=k(t)
$$

Finally, average human wealth, $h(t)$, is given by $h(t)=\int_{-\infty}^{t} l(v, t) h(v, t) d v=\beta h(t, t)$. Define $x(t) \equiv c(t) / k(t)$. We are now ready to represent a perfect foresight equilibrium by three differential equations:

$$
\begin{aligned}
g_{c}(t) \equiv \frac{\dot{c}(t)}{c(t)} & =\frac{r-\rho}{\sigma}+\frac{\hat{\sigma}}{\sigma}\left[\lambda-(b+\lambda) \frac{\Delta^{-1}(t)}{x(t)}\right] \\
g_{k}(t) \equiv \frac{\dot{k}(t)}{k(t)} & =(A-\delta)-x(t), \\
& \dot{\Delta}(t)=-1+\Delta(t)\left[r+d-\frac{r-\rho}{\hat{\sigma}}-\frac{\hat{\sigma}-\sigma}{\hat{\sigma}} g_{c}(t)\right] .
\end{aligned}
$$

If $(b, \lambda) \gg 0$, there is a continuous inflow of new cohorts without accumulated wealth $(b>0)$, and there is a continuous decay of human wealth of any existing cohort over time $(\lambda>0)$. As a consequence, the total wealth (human and accumulated) of a new cohort may differ from the average wealth, which gives rise to the generation replacement effect.

Generation Replacement Effect. The generation replacement effect refers to the difference between average and individual consumption growth rates, which is based on the fact that individual consumption levels change with age: $c(v, t) \neq c(t)$, but a household's individual consumption growth rate is independent of age. Analytically, the generation replacement 
effect — captured by the term $\Gamma(t)$ - is determined by the term in square brackets in (17):

Definition $1 \Gamma(t) \equiv g_{c}(t)-g_{c(v, .)}(t)=\frac{\sigma}{\hat{\sigma}} g_{c}(t)-\frac{r-\rho}{\hat{\sigma}}=\lambda-(b+\lambda) \frac{\Delta^{-1}(t)}{x(t)}$.

Two observations deserve remarks. First, if $\lambda=0$ (and $b>0$ ), the generation replacement effect is always negative $(\Gamma(t)<0)$. In this case, $h(v, t)=h(t)$. That is, a newborn's wealth is lower than average wealth: $a(t, t)+h(t, t)=h(t)<a(t)+h(t)$. Consequently, the consumption levels of young cohorts are lower than the average ones. As the individual consumption growth rate is independent of age, according to (15), and older cohorts are being constantly replaced by new cohorts without accumulated wealth. By the very fact that newborn cohorts — with consumption levels lower than the average consumption level - continuously enter the economy, the average consumption growth rate falls short of the individual consumption growth rate, $g_{c}(t)<g_{c(v, .)}(t)$.

Second, the sign of the generation replacement effect equals the sign of the difference between a newborn generation's total wealth and average total wealth: $\operatorname{sgn} \Gamma(t)=$ $\operatorname{sgn}[h(t, t)-[a(t)+h(t)]]$. If $\lambda$ is high enough so that $h(t, t)-[a(t)+h(t)]>0$, total wealth declines with age. As consumption is proportional to total wealth, $g_{c}(t)>g_{c(v, .)}(t)$.

As a consequence of the above remarks, there exists a strictly positive value for the retirement parameter, $\bar{\lambda}(t)$, such that $\Gamma(t)=0$. At a given date $t, \lambda \lessgtr \bar{\lambda}(t) \Leftrightarrow \Gamma(t) \lessgtr 0$. As the sign of $\Gamma(t)$ plays an important role in the results presented in the following section, we summarize these observations.

\section{Lemma 1}

(i) $\lambda=0 \Rightarrow \Gamma(t)<0$.

(ii) $\operatorname{sgn} \Gamma(t)=\operatorname{sgn}[h(t, t)-[a(t)+h(t)]]$.

(iii) If $b>0, \exists \bar{\lambda}(t)>0: \lambda \lessgtr \bar{\lambda} \Leftrightarrow \Gamma(t) \lessgtr 0$; if $b=0, \Gamma(t)=0$.

\section{Balanced Growth and the Desire to KUJ}

A balanced growth path (BGP) is defined by constant growth rates: $g_{k}=g_{y}=g_{c}=g$, and $\dot{\Delta}=0$. Notice that, depending on the sign of the generation replacement effect, $g_{c}$ is 
generally different from $g_{c(v, .)}$. As all growth rates are constant, we omit time indexes as of here. From (15) it follows:

$$
\Gamma=-\frac{r-\rho-\sigma g}{\hat{\sigma}}=\underset{+}{(g, \hat{\sigma})}
$$

For a given growth rate, $g, \Gamma$ declines (rises) in $\hat{\sigma}$ if $\Gamma>0$ (if $\Gamma<0$ ). We can now characterize a BGP in the variables $(x, g)$ :

$$
\begin{aligned}
& x^{c}=(b+\lambda) \frac{(r+d-g)+\Gamma(g, \hat{\sigma})}{\lambda-\Gamma(g, \hat{\sigma})}, \\
& x^{k}=(A-\delta)-g,
\end{aligned}
$$

where $x^{c}=\left.x\right|_{g_{c}=g}, x^{k}=\left.x\right|_{g_{k}=g}$, and we consider that $\Delta^{-1}=r+d-\frac{r-\rho}{\hat{\sigma}}-\frac{\hat{\sigma}-\sigma}{\hat{\sigma}} g$. Clearly, a BGP satisfies $x^{c}=x^{k}=x$. Notice that $\partial x^{c} / \partial g>0$, and $\partial x^{k} / \partial g<0$. Given $g<(A-\delta)$, a BGP exists if $\left.x_{c}\right|_{g=0}<A-\delta$. Equating (21) and (22) yields a quadratic equation in $g$, whose smaller root is: ${ }^{6}$

$$
\begin{aligned}
& g=\frac{B}{2 \sigma}-\frac{\sqrt{B^{2}-4 \sigma[(A-\delta)[r-\rho+\hat{\sigma} \lambda]-(b+\lambda)[\hat{\sigma}(r+d)-(r-\rho)]]}}{2 \sigma}, \\
& \text { where } B \equiv r-\rho+\hat{\sigma} \lambda+(A-\delta) \sigma-(b+\lambda)(\hat{\sigma}-\sigma)
\end{aligned}
$$

It is important to observe that the generation replacement effect has a second interpretation. It represents the intergenerational consumption growth rate. As shown in the appendix:

$$
\frac{c_{v}(v, .)}{c(v, .)}=g-g_{c(v, .)}=\Gamma
$$

At any given date $t$, individual consumption grows with age when $\Gamma<0$, and it declines with age when $\Gamma>0$. If $\Gamma<0$, a new cohort's total wealth, $h(t, t)$ is lower than average total wealth, $a(t)+h(t)$. Consequently $c(t, t)<c(t)$, and consumption rises in age. The reverse holds when $\Gamma>0$.

\subsection{The Desire to KUJ}

We now investigate the impact of the desire to KUJ on the balanced growth rates.

\footnotetext{
${ }^{6}$ The larger root is ruled out by the requirement: $g<r+d+\lambda$. To see this, differentiate $h(t)$ with respect to time. For a BGP, it holds: $h / y=\beta(1-\alpha) /(r+d+\lambda-g)$, which is required to be positive.
} 
Proposition 1 The impact of the desire to KUJ on the balanced growth rates of consumption and output depends on the sign of the generation replacement effect. In particular:

$$
\Gamma \lessgtr 0 \Leftrightarrow \frac{\partial g}{\partial \eta} \lessgtr 0
$$

The proof is given in the appendix. From (22) it follows that the impact of the desire to KUJ on growth, $g$, is opposite to that on the propensity to consume out of accumulated wealth, $x$. Upon a rise in the reference parameter, either, the propensity to consume is increased - at the cost of a lower consumption growth rate. Or, the propensity to consume is decreased - at the benefit of a higher consumption growth rate. This observation displays an intertemporal trade-off as regards the desire to KUJ. Consuming more today rises one's consumption relative to the reference level, ceteris paribus. If $\eta>0$, additional utility is gained from a rise in $c(t, t) / c(t)$. This benefit comes at a cost, however. Consuming more today lowers tomorrow's consumption possibilities, thereby tomorrow's consumption relative to the reference level, ceteris paribus.

The proposition shows that the benefit exceeds the cost if $\Gamma<0$. In this case, initially, for a given wealth level, a rise in $\eta$ raises individual consumption levels - that is the propensity to consume out of accumulated wealth — and, thereby, lowers the individual consumption growth rate. Consequently, along the new BGP (with a higher level of the reference parameter), the propensity to consume out of accumulated wealth is higher and the growth rate is lower than along the initial BGP.

The situation is the opposite if $\Gamma>0$. In this case, the benefit - regarding the desire to KUJ - of (initially) raising the consumption level is lower than the cost of suffering from a lower consumption growth rate (thereby from lower individual future consumption levels relative to future reference consumption).

As a consequence, it is mistaken to assume that the desire to KUJ inevitably leads to higher growth rates of consumption. At the same time, it is mistaken to assume that the desire to KUJ inevitably leads to a higher propensity to consume out of accumulated wealth. As long as $\Gamma \neq 0$, however, one of the two possibilities will apply. Figure 1 depicts the result offered by Proposition 1 . 


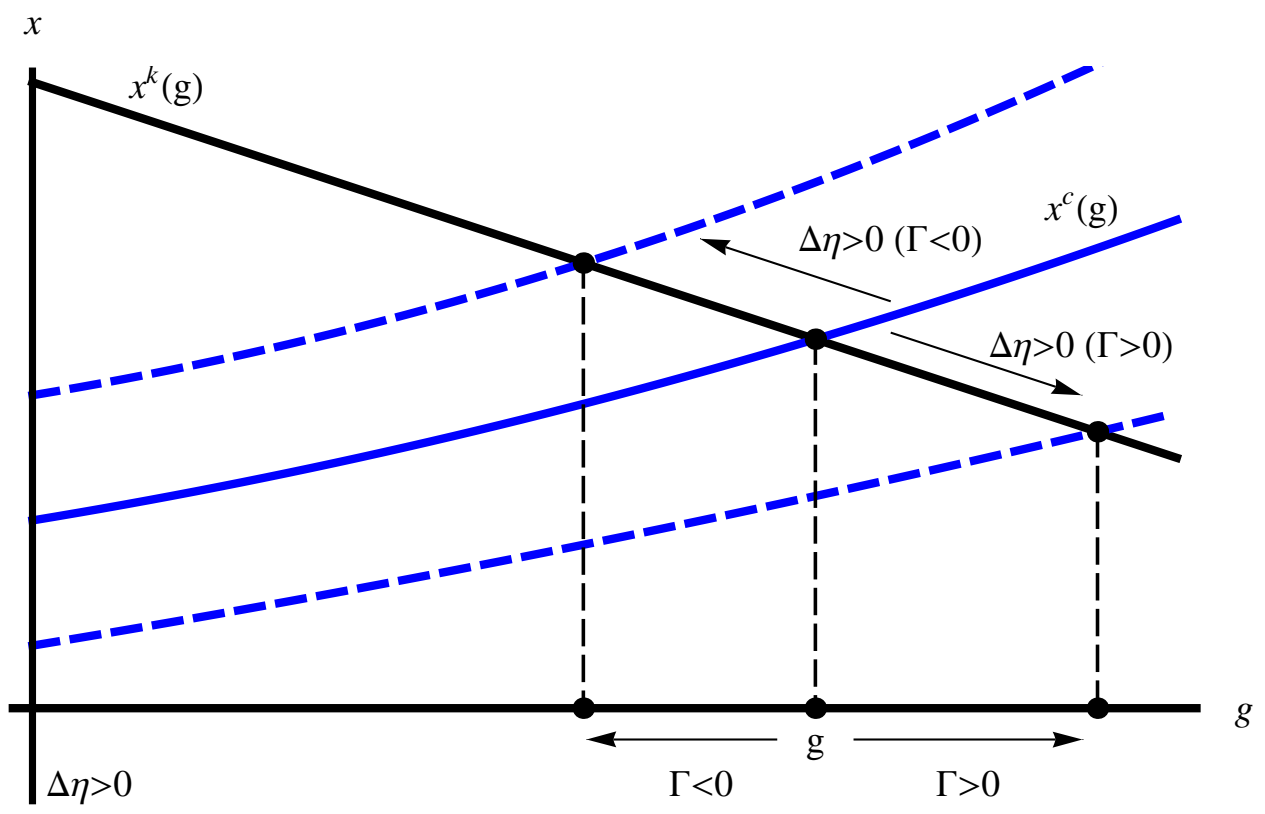

Figure 1: Impact of the Desire to KUJ on $(g, x)$

Corollary 1 Suppose $b>0$ and $\lambda=\bar{\lambda}$. Then the desire to KUJ does not affect the balanced growth rates of consumption and output.

According to Lemma 1, if $\lambda=\bar{\lambda}, h(t, t)=h(t)+a(t)$, and $g_{c}=g_{c(v, .)}$. That is, individual and average consumption growth rates coincide. In this knife-edge case, the benefit of consuming more today (relative to the reference level) exactly offsets the cost of lowering the consumption growth rate. Consequently, in this knife-edge case, a marginal rise in the reference parameter has no impact on the balanced growth rates of consumption and output.

Corollary 2 Suppose $b=0$. Then the desire to KUJ does not affect the balanced growth rates of consumption and output.

In the proof of Lemma 1 (see appendix), it was shown that $b=0$ implies that the generation replacement effect equals zero. Thus, with exogenous labor supply, a rise in the reference parameter has no impact on the balanced growth rates of consumption and output. $^{7}$ This result was demonstrated for representative agent economies before (Turnovsky

\footnotetext{
${ }^{7}$ It is important to note that this result is true only if the marginal rate of substitution of individual consumption for reference consumption is constant in time along the BGP (see Turnovsky and Monteiro
} 
and Monteiro, 2007). Clearly, the case of $b=0$ yields the representative agent economy as a special case of the continuous time overlapping generations framework.

Proposition 1 has its foundation in the response of individual households to a rise in the reference parameter.

Proposition 2 The impact of the desire to KUJ on the balanced growth rate of individual consumption depends on the sign of the generation replacement effect. In particular:

$$
\Gamma \lessgtr 0 \Leftrightarrow \frac{\partial g_{c(v, .)}}{\partial \eta} \lessgtr 0 .
$$

A rise in $\eta$ raises the (absolute value of the) elasticity of marginal utility with respect to individual consumption. If $\Gamma<0$, a rise in $\eta$ induces the marginal utility to decline too strongly over time. Households aim to lower their consumption growth rates. Consequently, households bring some future consumption forward to the present and lower their consumption growth rates. As, initially, every individual household raises its consumption level, average consumption rises as well. This reaction, in turn, implies a lowering in aggregate savings. Subsequently the capital stock declines, and the new steady state is characterized by a lower average propensity to consume out of accumulated wealth, $x$.

The response is the opposite if $\Gamma>0$, in which case, $g_{c(v, .)}<g$. A rise in $\eta$ induces the marginal utility to rise too strongly over time, and households aim to raise their consumption growth rates. Consequently, households will shift consumption from the present to the future.

The key to this result is the sign of the generation replacement effect. If $\Gamma<0$, $g_{c(v, .)}>g$, and individual households respond to a rise in $\eta$ with a rise in the propensity to consume, $x$ (rather than with a rise in $g_{c(v, .)}$ ). If $\Gamma>0, g_{c(v, .)}<g$, and individuals respond to a rise in $\eta$ with a rise in consumption growth (rather than with a rise in $x$ ).

If $\lambda=0$, then $\Gamma<0$, and the desire to KUJ always raises the propensity to consume out of total wealth and lowers the growth rate of consumption and output in a BGP. This is true for all $\lambda<\bar{\lambda}$. However, once $\lambda>\bar{\lambda}$, the desire to KUJ lowers the propensity to 2007). 
consume out of total wealth and raises the growth rates of consumption and output in a BGP.

Considering Propositions 1 and 2, the natural question to ask is whether or not $\Gamma$ is likely to be positive. Using one year as our time unit, we can obtain a rough estimate of $\Gamma$. The following parameters have to be assigned values: $(\bar{A}, b, \alpha, \delta, \varepsilon, \eta, \lambda, \rho, \sigma)$. In accordance with usual numerical estimates, $\alpha=1 / 3, \delta=0.1, \rho=0.018, \sigma=2$. The values of parameters $\alpha$ and $\delta$ are the usual values for capital depreciation and for the output elasticity with respect to private capital accumulation. Likewise, the values of the preference parameters $(\rho, \sigma)$ accord well with empirical studies. Less information is available for parameters $(\varepsilon, \eta)$. Wendner and Goulder (2008) demonstrate that a reasonable range for $\eta$ is: $\eta \in[0.2,0.4]$. For our numerical simulation, we choose $\eta=0.2$. The scale effect parameter can be shown to have only a minor impact on numerical simulations, once $n=0$. We choose $\varepsilon=0.3$. In the present framework, the birth and death rates are equal. As $b=d$, a value of $b=d=0.02$ corresponds to an expected lifetime of an adult of about 50 years. Parameter values for $(\lambda, \bar{A})$ are still missing. First, an estimate for the labor force participation rate is $\beta=0.75$. Given $\beta=b /(b+\lambda)$, we conclude, $\lambda=0.007$. Given this value for $\lambda$, we can calculate a value for $\bar{A}$ that is consistent with a reasonable rate of interest and investment share: $\bar{A}=1 / 2$.

This calibration implies the following: $g=0.02, r=0.057, c / y=0.74$. Moreover, $\bar{\lambda}=0.004$. That is, $\lambda$ greatly exceeds $\bar{\lambda}$, and $\Gamma=0.002>0$. For a reasonable calibration of the model, the generation replacement effect is positive. In this case, Propositions 1 and 2 imply that the desire to KUJ raises the balanced growth rates and lowers the average propensity to consume out of accumulated wealth.

\subsection{Optimal Balanced Growth and the Desire to KUJ}

Once market prices fail to reflect to social value of capital, the market mechanism may yield a rate of capital accumulation that is not optimal. The present framework exhibits two sources for market failure: a production externality (stemming from investment), and a consumption externality (the desire to KUJ). The desire to KUJ was shown to either raise 
or lower the balanced growth rates, depending on the sign of the generation replacement effect. Thus, a priori, it is not clear, whether or not the balanced growth rates in the market economy are lower than optimal.

We characterize a utilitarian optimal balanced growth allocation, following Calvo and Obstfeld (1988), Willenbockel (2008), and Wendner (2009). The time-consistent utilitarian social welfare function takes the form:

$$
W(t)=\int_{-\infty}^{t} L(v, t) U(v, t) e^{-\rho(t-v)} e^{-\tilde{\rho}(v-t)} d v+\int_{t}^{\infty} L(v, v) U(v, v) e^{-\tilde{\rho}(v-t)} d v,
$$

where $\tilde{\rho}$ is the social planner's discount rate. In the following, we assume that the pure rate of time preference and the social planner's discount rate, $\tilde{\rho}$, coincide:

$$
\tilde{\rho}=\rho .
$$

Under the assumptions of $n=0$ (as above), and $\tilde{\rho}=\rho$, the welfare function becomes:

$$
W(t)=\int_{t}^{\infty}\left\{\int_{-\infty}^{s} b u[c(v, s), c(s)] e^{-b(s-v)} d v\right\} e^{-\rho(s-t)} d s .
$$

As from here, we indicate optimal values by a tilde. Notice that $\tilde{r}=A-\delta$, while $r=\alpha A-\delta$. As $\rho=\tilde{\rho}$, the social planner treats all generations alike: $\tilde{c}(v, t)=\tilde{c}\left(v^{\prime}, t\right)$. This implies: $\tilde{c}(t)=\tilde{c}(v, t)$. Two immediate consequences are: there is no generation replacement effect: $\Gamma=0 ;$ and $\tilde{g}_{c}=\tilde{g}_{g_{c(v, .)}}$. The optimal BGP is described by:

$$
\begin{aligned}
\tilde{g}_{c(v, .)} & =\frac{\tilde{r}-\rho}{\hat{\sigma}}+\frac{\hat{\sigma}-\sigma}{\hat{\sigma}} \tilde{g}, \\
\tilde{g} & =\tilde{g}_{c(v, .)}=\frac{\tilde{r}-\rho}{\sigma}, \\
\tilde{x} & =(A-\delta)-\tilde{g} .
\end{aligned}
$$

Comparing the market economy's BGP with the optimal one yields:

Proposition 3 At a BGP, the optimal growth rate, $\tilde{g}$, may exceed or fall short of the market economy's growth rate $\mathrm{g}$. In particular:

$$
g-\tilde{g} \gtrless 0 \Leftrightarrow \frac{r-\tilde{r}+\hat{\sigma} \Gamma}{\sigma} \gtrless 0 .
$$

Consequently, if $\Gamma \leq 0, g<\tilde{g}$. 
Once market prices fail to reflect the social value of capital, the market mechanism may yield a rate of capital accumulation - thereby a balanced growth rate of output and average consumption - that is lower than optimal. As long as $\Gamma<0$, this intuition is confirmed by the present framework with AK technology and a KUJ consumption externality. As $r<\tilde{r}, \Gamma \leq 0$ implies $g<\tilde{g}$.

The desire to KUJ strengthens the difference between $g$ and $\tilde{g}$. According to Proposition 1, if $\Gamma<0$, the desire to KUJ lowers the market economy's balanced growth rate. The optimal growth rate, however, is not affected by the desire to KUJ, according to (28). ${ }^{8}$

That the desire to KUJ lowers the balanced growth rate relative to the optimal one can equivalently be stated by the observation that the desire to KUJ raises the average propensity to consume out of accumulated wealth, $x$, relative to the optimal propensity: $x>\tilde{x} \cdot{ }^{9}$ In the pursuit to KUJ, households raise their consumption (more than optimal), hence, lower their savings. It follows that the propensity to consume is increased.

If $\Gamma<0$, growth enhancing policies enhance social welfare for two reasons. First, for a given rate of interest, savings are too low as households overconsume in the pursuit to KUJ. Second, firms underinvest, as the private rate of interest fails to reflect the social value of capital. Both externalities contribute to a lower than optimal balanced growth rate.

The optimal growth enhancing policy, along a BGP, involves two instruments: a (negative) capital income tax, and a system of personalized lump sum transfers (and taxes). The capital income tax rate, $\tau_{k}$, is required to equalize the social and private rates of return to capital: $r\left(1-\tau_{k}\right)=\tilde{r} \Rightarrow \tau_{k}=-(1-\alpha) A /(\alpha A-\delta)<0$. That is, capital accumulation must be subsidized. The optimal capital income subsidy is necessary to ensure that the market economy's intertemporal balanced growth rates are optimal. Clearly, the desire to KUJ has no impact on the optimal capital income subsidy.

The program of lump sum transfers ensures that the market economy's intergenerational growth rate is optimal (that is, zero, as private and social discount rates coincide).

\footnotetext{
${ }^{8}$ This result holds true when the desire to KUJ is represented by the marginal degree of positionality, as proposed by Johansson-Stenman et al. (2002).

${ }^{9}$ This follows directly from: $x=(A-\delta)-g$ in both the market economy and the optimal framework.
} 
Let $t=0$ be the date of introduction of the optimal tax program. The intertemporal program of lump sum taxes and transfers must be such that for each individual household, the present value of the respective lump sum tax-/transfer stream leads the household to consume an amount equal to $\tilde{c}(0)$. That is: $c(v, 0)=c(0)=\tilde{c}(0)=\tilde{c}(v, 0), v \leq 0$. Such a program can be implemented as a balanced budget tax-/transfer scheme for all $t \geq 0$, as argued in the appendix. Then, at $t=0$, all individual consumption levels are equal (and optimal). For a given and optimal $k(0), c(0)$ is optimal as well. Moreover, as $c(0,0)=c(0)$, $\Gamma=0$ implying: $g_{c(v, .)}(0)=g(0)=\tilde{g}(0)$, where the last equality follows from the optimal capital income subsidy.

If $\Gamma<0$, in a market economy without taxes and transfers, $c(t, t)<c(t)$. Thus, the optimal lump sum transfer program must redistribute resources from older to younger cohorts. We call such a tax program "progressive" in an intergenerational sense; the tax burden increases with age. Likewise, if $\Gamma>0, c(t, t)>c(t)$, and the optimal transfer program must redistribute from the young to the elder generations. We call such a tax program "regressive" in an intergenerational sense; the tax burden decreases with age. Let $\tau(v, t)$ be the present value of the stream of lump sum transfers or taxes of a vintage- $v$ household at date $t$, where $\tau(v, t)>0(\tau(v, t)<0)$ indicates that the household receives a transfer (pays a lump-sum tax) in present value. Also, let $\tau(t) \equiv \int_{-\infty}^{t} l(v, t) \tau(v, t) d v$. Progressivity (regressivity) of the lump sum transfer system is then defined by:

Definition 2 A lump sum transfer system is said to be progressive if: $\left[\frac{\tau(t)}{k(t)}-\frac{\tau(t, t)}{k(t)}\right]<0$. A lump sum transfer system is said to be regressive if: $\left[\frac{\tau(t)}{k(t)}-\frac{\tau(t, t)}{k(t)}\right]>0$.

That is, progressivity either means that the youngest generation receives a transfer, while the average pays a lump sum tax, in present value, $\tau(t, t)>0$ and $\tau(t)<0$, or both generations pay a lump sum tax, but the average burden is higher than the youngest generation's burden. Likewise, regressivity either means $\tau(t, t)<0$ and $\tau(t)>0$, or $\tau(t, t)<0$ and $\tau(t)<0$ where the average burden is lower than the youngest generation's burden.

Proposition 4 (Optimal Tax Program) 
(i) $\operatorname{sgn}[\tau(t)-\tau(t, t)]=\left.\operatorname{sgn} \Gamma(t)\right|_{\tau(t, t)=\tau(t)=0}$.

(ii) $\left.\Gamma(t)\right|_{\tau(t, t)=\tau(t)=0}<0 \Rightarrow \tau(t)<0$ and $\tau(t, t) \gtrless 0 \Leftrightarrow \tau_{k} \gtrless \tau_{k}^{m}<0$,

$\left.\Gamma(t)\right|_{\tau(t, t)=\tau(t)=0}>0 \Rightarrow \tau(t, t)<0$, and $\tau(t) \gtrless 0 \Leftrightarrow \tau_{k} \gtrless \tau_{k}^{m^{\prime}}<0$,

for well defined lower bounds on $\tau_{k}$.

The proof ${ }^{10}$ of Proposition 4 is given in the appendix. It specifies the lower bounds on $\tau_{k}, \tau_{k}^{m}$ and $\tau_{k}^{m^{\prime}}$. The proposition shows that an optimal lump sum transfer program is progressive (regressive) when $\Gamma<0(\Gamma>0)$. For obtaining an optimal (egalitarian, as $\rho=\tilde{\rho}$ ) intergenerational allocation, the optimal lump sum transfer program redistributes resources from wealthy to poor generations.

A slight complication arises from the need to finance the optimal capital income subsidy. As long as $\tau_{k}>\max \left\{\tau_{k}^{m}, \tau_{k}^{m^{\prime}}\right\}$, the optimal lump sum transfer program imposes a tax on the wealthy generations and grants a transfer on the poor generations. However, once $\tau_{k}<\min \left\{\tau_{k}^{m}, \tau_{k}^{m^{\prime}}\right\}$, the revenue requirement for financing the optimal capital income subsidy becomes so dominant that all generations have to pay lump sum taxes. In this case, Proposition 4 implies that the elder (younger) generations carry the major burden of the lump sum transfer program when $\Gamma<0(\Gamma>0)$.

Proposition 5 Suppose, $\Gamma<0$. At a BGP, the desire to KUJ raises the progressivity of the optimal intertemporal lump sum tax-/transfer program. In particular, $\left[\frac{\tau(t)}{k(t)}-\frac{\tau(t, t)}{k(t)}\right]$ declines (becomes more negative) in $\eta$.

The desire to KUJ raises the (absolute) of the elasticity of marginal utility with respect to average consumption: $\hat{\sigma}_{\eta}>0$. As a consequence, the individual consumption growth rate is reduced (see Proposition 2), otherwise, marginal utility would decline at too big a rate.

Intuition can be gained by the following consideration. As $\Gamma<0$, total (accumulated and human) individual wealth rises with age. Consequently, at any given point in time, individual consumption rises with age. Every household lowers its consumption growth rate by the same amount - that is, every household raises its consumption level proportionally

\footnotetext{
${ }^{10}$ The proof also argues that the optimal tax program can be implemented with a balanced government budget at every date.
} 
to its initial consumption level. Thus, the youngest cohort entering the economy raises its consumption level by the smallest (that is, a below average) amount. As a consequence, the ratio $c(t, t) / c(t)$ declines and so does $\Gamma=b[c(t, t) / c(t)-1]$. The desire to KUJ leads the consumption profile to become (even) steeper with age. ${ }^{11}$ The optimal tax program responds to the steepening of the intergenerational progressivity with a stronger redistribution from old (wealthy) to young households.

Finally, we turn to the case with a positive generation replacement effect. With the optimal capital income tax in place, if $\Gamma>0, r\left(1+\tau_{k}\right)-\tilde{r}+\hat{\sigma} \Gamma=\hat{\sigma} \Gamma>0$. Proposition 3 then implies that the market economy's balanced growth rate exceeds the optimal one: $g>\tilde{g}$. Moreover, the desire to KUJ further raises the market economy's balanced growth rate, according to Proposition 1. In this case, a growth enhancing policy is mistaken, and it diminishes social welfare.

Proposition 6 Suppose, $\Gamma>0$. At a BGP, the desire to KUJ raises the (intergenerational) regressivity of the optimal intertemporal lump sum tax-/transfer program. In particular, $\left[\frac{\tau(t)}{k(t)}-\frac{\tau(t, t)}{k(t)}\right]$ rises in $\eta$.

If $\Gamma>0$, both total (accumulated and human) individual wealth and individual consumption levels decline with age. As above, the desire to KUJ raises the (absolute) of the elasticity of marginal utility with respect to average consumption. That is, a given negative individual consumption growth rate lets a household's marginal utility increase at too big a rate. Therefore households respond by raising the individual consumption growth rate (that then becomes less negative).

As all households raise their consumption growth rate by the same percentage, wealthy (young) households raise their consumption levels at an above average extent. Consequently, the ratio $c(t, t) / c(t)$ increases, and so does $\Gamma$. The the consumption profile declines more strongly with age. The optimal tax program responds to the steepening of the intergenerational regressivity with a stronger redistribution from young (wealthy) to old households.

\footnotetext{
${ }^{11}$ That is, $\Gamma=g-g_{c(v, .)}=c_{v}(v, t) / c(v, t)$, and $\Gamma_{\eta}<0$.
} 
Propositions 5 and 6 are related to the curvature of the utility function. The utility function is strictly concave. Roughly, with the wealth (consumption) profile increasing with age, the desire to KUJ - making the utility function more concave - requires a shift of individual consumption to the period with a low total individual wealth. Hence, households raise their propensity to consume (they consume more earlier at life), and they lower their consumption growth rate (they consume less later in life). The opposite is true when the wealth (consumption) profile decreases with age. In this case, a household gains from shifting consumption from the present to the future - that is, from lowering the propensity to consume out of total wealth and, thereby, increasing its consumption growth rate.

Proposition 6 has two important implications. First, if $\Gamma>0$, an unfunded social security system — as is in place in many countries — rises social welfare (independently from the desire to KUJ). However, an unfunded social security system would lower social welfare if $\Gamma<0$, in which case welfare would be improved by a "reverse unfunded social security system." As a main conclusion, in order to be able to assess the welfare impact of an unfunded social security system (such as a pay as you go pension system), the sign of $\Gamma$ matters.

Second, the desire to KUJ justifies an intergenerational "strengthening" of the optimal social security system. If $\Gamma<0$, the desire to KUJ justifies a strengthening of the progressivity of the optimal tax system. More funds need to be redistributed from the old to the young. If $\Gamma>0$, the desire to KUJ justifies a strengthening of the regressivity of the optimal tax system. More funds need to be redistributed from the young to the old.

\section{Conclusions}

This paper investigates the effects of the desire to KUJ on balanced growth and optimal tax policy in the framework of a continuous time overlapping generations economy with gradual retirement, exogenous labor supply, and AK technology. The analysis yields two main conclusions.

First, the effects of the desire to KUJ on a household's propensity to consume out of 
total wealth, and its balanced consumption growth rate depend on whether individual total (accumulated and human) wealth is increasing or decreasing with age. If total individual wealth is increasing with age, the desire to KUJ raises the propensity to consume out of total wealth and lowers the individual consumption growth rate. The reverse is true, however, when individual total wealth is decreasing with age.

The main reason was seen to stem from concavity of the utility function. The desire to KUJ makes the utility function more concave. Consequently, households shift consumption to their low wealth-period, which is early in life for a rising wealth profile and late in life for a declining wealth profile. In other words, individual households raise their balanced consumption growth rate if wealth is declining with age, otherwise they reduce their balanced consumption growth rate.

The rate of retirements determines whether or not individual total wealth rises with age. If the rate of retirement is large enough, the individual total wealth decreases with age, otherwise it increases with age. Clearly, there exists a specific rate of retirement for which the total wealth is constant with age. In this knife-edge case, the desire to KUJ has no impact on the balanced growth rates.

Second, the above positive findings are reflected in an optimal tax program. Two instruments are needed in order to decentralize an optimal allocation. A lump sum transfer program ensures optimality of the intergenerational consumption growth rate at every date $t$. A subsidy on capital income ensures optimality of the intertemporal consumption growth rate.

The lump sum transfer system involves intergenerational redistribution. The redistribution goes from old to young when total individual wealth increases with age, and it goes from young to old when total individual wealth decreases with age. In both cases, the desire to KUJ raises the optimal levels of intergenerational redistribution, which becomes more progressive (regressive) when individual wealth increases (decreases) with age.

Key to these results is the difference between individual and average consumption growth rates, termed "generation replacement effect" in the present study. The sign of the generation replacement effect determines whether or not the balanced growth rates rise due 
to the desire to KUJ. A rough numerical simulation shows that the generation replacement effect may well be positive. However, considerable scope remains for developing better estimates for the generation replacement effect. One task for future research then is to identify the conditions for which the sign of the generation replacement effect is positive (or negative).

\section{Appendix}

Derivation of Individual and Average Consumption. For any individual, consider the current value Hamiltonian:

$$
H_{c}=u(c(v, \tau), c(\tau))+\mu(\tau)[(r+d) a(v, \tau)+w(v, \tau)-c(v, \tau)]
$$

For all $\tau \geq t \geq v, c(v, \tau)$ is chosen such as to maximize expected utility (11), subject to:

$$
\begin{aligned}
& c(v, \tau) \geq 0, \\
& \dot{a}(v, \tau)=(r+d) a(v, \tau)+w(v, \tau)-c(v, \tau), \\
& a(v, t) \text { given, } \lim _{\tau \rightarrow \infty} \mu(\tau) e^{-(\rho+d) \tau} a(v, \tau)=0 .
\end{aligned}
$$

From $\partial H_{c} / \partial c(v, \tau)=0$ and $-\partial H_{c} / \partial a(v, \tau)=\dot{\mu}-(\rho+d) \mu$, it follows:

$$
g_{c(v, .)}(\tau)=\frac{\dot{c}(v, \tau)}{c(v, \tau)}=\frac{r-\rho}{\hat{\sigma}}+\frac{\hat{\sigma}-\sigma}{\hat{\sigma}} \frac{\dot{c}(\tau)}{c(\tau)}
$$

where no individual considers its impact of individual consumption on the reference level. Thus,

$$
c(v, \tau)=c(v, t) e^{\int_{t}^{\tau} g_{c(v, .)}(s) d s} .
$$

Combining this equation with the intertemporal budget constraint (12), and considering the definition of $\Delta(t)$ in (13) yields: $c(v, t)=\Delta^{-1}(t)[a(v, t)+h(v, t)]$, and $c(t, t)=$ $\Delta^{-1}(t) h(t, t)=\Delta^{-1}(t) \beta^{-1} h(t)$.

Considering $c(t)=\Delta^{-1}(t)[a(t)+h(t)]$, the growth rate of average consumption follows from: $\dot{c}(t)=b c(t, t)-b c(t)+g_{c(v, .)}(t) c(t) . \|$ 


\section{Proof of Lemma 1.}

(i) From the definition of $\Gamma(t)$ it follows: $\left.\Gamma(t)\right|_{\lambda=0}=-b \frac{\Delta^{-1}(t)}{x(t)}<0$.

(ii) As $\Delta^{-1}(t)=c(t) /[h(t)+a(t)], \Gamma(t)=\lambda-(b+\lambda) \frac{\Delta^{-1}(t)}{x(t)}=\lambda-(b+\lambda) a(t) /[a(t)+h(t)]$. Thus, $\Gamma(t)[a(t)+h(t)] / b=[(\lambda / b) h(t)-a(t)]$. The sign of the term on the right hand side equals the sign of $\Gamma(t)$. At the same time, the difference between a new cohort's and average total wealth equals: $h(t, t)-h(t)-a(t)=h(t) / \beta-h(t)-a(t)=[(\lambda / b) h(t)-a(t)]$.

(iii) Suppose $b=0$. It follows: $\Gamma(t)=b[c(t, t)-c(t)] / c(t)=0$.

Suppose $b>0 .[c(t, t)=c(t)] \Leftrightarrow[b / \lambda=h / k]$, where $h / k=(1-\alpha) A /[r+d+\lambda-g]>0$, along a BGP. Solving for $\lambda$ yields: $\bar{\lambda}=b(r+d-g(\bar{\lambda})) /[(1-\alpha) A(\bar{\lambda})-b]$, which implicitly defines $\bar{\lambda}$. $\|$

Intergenerational Consumption Growth. We assume $0<v<t$. From $c(v, t)=$ $\Delta^{-1}(t)[a(v, t)+h(v, t)]$ and $c(v, v)=\Delta^{-1}(t) h(v, v)$ it follows, for a BGP:

$$
c(v, t)=c(v, v) e^{g_{c(v, .)}(t-v)}=\Delta^{-1}(t) h(v, v) e^{g_{c(v, .)}(t-v)}=\Delta^{-1}(t) \beta^{-1} h(0) e^{g v} e^{g_{c(v, .)}(t-v)} .
$$

Considering that both $g_{c(v, .)}$ and $h(0)=w(0) /[r+d+\lambda-g]$ are independent of $v$ :

$$
c(v, t)=\Delta^{-1}(t) \beta^{-1} h(0) e^{\left(g-g_{c(v, .)}\right)} e^{g_{c(v, .)} t} .
$$

Consequently, $c_{v}(v, t)=\left[g-g_{c(v, .)}\right] c(v, t) . \|$

Proof of Proposition 1. In $(g, x)$ space, the $x^{k}$-curve is downward sloping, while the $x^{c}$-curve is upward sloping. We consider the shifts of the $x^{c}$ - and $x^{k}$-curves upon a rise in $\eta$. A rise in $\eta$ has no impact on the $x^{k}$-curve. From (21) it follows that for a given $g$, $\partial x^{c} /(\partial \Gamma)>0$. Moreover, $\partial \Gamma /(\partial \hat{\sigma}) \gtreqless 0 \Leftrightarrow \Gamma \lesseqgtr 0$. Also, $\partial \hat{\sigma} /(\partial \eta)>0$.

(i) If $\Gamma<0$, a rise in $\eta$ raises $\hat{\sigma}$, which raises $\Gamma$ and shifts the $x^{c}$-curve up. Consequently, $x$ increases and $g$ declines.

(ii) If $\Gamma>0$, a rise in $\eta$ raises $\hat{\sigma}$, which lowers $\Gamma$ and shifts the $x^{c}$-curve down. Consequently, $x$ declines and $g$ rises. $\|$ 
Proof of Proposition 2. Notice that $\hat{\sigma}_{\eta}>0$. From the definition of the generation replacement effect, $g_{c(v, .)}=g(\hat{\sigma})-\Gamma(\hat{\sigma})$. Thus:

$$
g_{c(v, .)}^{\prime} \equiv \frac{\partial g_{c(v, .)}}{\partial \hat{\sigma}}=g^{\prime}(\hat{\sigma})-\Gamma^{\prime}(\hat{\sigma})
$$

where $g^{\prime}(\hat{\sigma}) \equiv \partial g() /.(\partial \hat{\sigma})$ and $\Gamma^{\prime}(\hat{\sigma}) \equiv \partial \Gamma() /.(\partial \hat{\sigma})$. Considering $\Gamma=-[r-\rho-\sigma g(\hat{\sigma})] / \hat{\sigma}$, $\Gamma^{\prime}(\hat{\sigma})=\left[-\Gamma(\hat{\sigma})+\sigma g^{\prime}(\hat{\sigma})\right] / \hat{\sigma}$. Thus:

$$
g_{c(v, .)}^{\prime}=\frac{\Gamma}{\hat{\sigma}}+\frac{\hat{\sigma}-\sigma}{\hat{\sigma}} g^{\prime}(\hat{\sigma}) .
$$

We observe that $\hat{\sigma}-\sigma \geq 0$, and $\hat{\sigma}_{\eta}>0$. From Proposition 1 , if $\Gamma(\hat{\sigma})<0, g^{\prime}(\hat{\sigma})<0$. Likewise, if $\Gamma(\hat{\sigma})>0, g^{\prime}(\hat{\sigma})>0$. Proposition 2 follows. $\|$

Proposition 3. Considering (15), (27), and Definition 1, it follows:

$$
g-\tilde{g}=\left[\frac{r-\rho}{\hat{\sigma}}-\frac{\tilde{r}-\rho}{\hat{\sigma}}\right]+\Gamma+\frac{\hat{\sigma}-\sigma}{\hat{\sigma}}(g-\tilde{g}) .
$$

From reordering terms, Proposition 3 immediately follows. ||

Proposition 4. At every date, every household receives a lump sum transfer $t(v, t)>0$ (or pays a lump sum tax $t(v, t)<0$ ). Let $\tau(v, t)$ be the present value (discounted at the annuity rate of interest, $r+d$ ) of the stream of lump sum taxes or transfers of a vintage- $v$ household at date $t$. Without loss of generality, we only consider programs with balanced budgets in every period. A balanced government budgets requires $\int_{-\infty}^{t} l(v, t) t(v, t) d v-$ $\tau_{k} r k(t)=0$ for all $t$. Consider the intertemporal budget constraint with $\pi(v, s)=t(v, s)-$ $r \tau_{k} a(v, s)$ :

$$
\begin{aligned}
& \int_{-\infty}^{t} \int_{t}^{\infty} \pi(v, s) e^{-R^{a}(t, s)} d s l(v, t) d v \\
+ & \int_{t}^{\infty} \int_{v}^{\infty} \pi(v, s) e^{-R^{a}(v, s)} d s l(v, v) e^{-r(v-t)} d v=0 .
\end{aligned}
$$

By the arguments offered by Calvo and Obstfeld (1988, p. 175), it can be easily shown that (30) holds (every household respects its budget constraint, so the government bud- 
get constraint is implied to hold). Given there is no initial government debt, the main consequence of (30) is that the present value of all primary deficits equals zero.

Now, consider any feasible optimal tax program with a date- $t$ primary deficit: $\pi(t)=\int_{-\infty}^{t} l(v, t) t(v, t) d v-\tau_{k} r k(t)$. The intertemporal government budget constraint (30) implies: $\int_{t}^{\infty} \pi(s) e^{-r(s-t)} d s=0$. The stream of all primary deficits can be added to the cohort born at $t=0$, without altering the present wealth of its lump sum transfer stream. Thus, the cohort does not change its consumption decision, while the government runs a balanced budget.

Next, along a balanced growth path, $\tau(v, v)$ grows at a constant rate, say $\gamma<r$ : $\tau(v, v)=\tau(t, t) e^{\gamma(v-t)}$. Considering a BGP, the government budget constraint can be written as:

$$
\frac{\tau(t)}{k(t)}=-\frac{b}{r-\gamma} \frac{\tau(t, t)}{k(t)}+\int_{t}^{\infty} r \tau_{k} e^{-(r-g)(s-t)} d s=-\frac{b}{r-\gamma} \frac{\tau(t, t)}{k(t)}+\frac{r \tau_{k}}{r-g} .
$$

Along a BGP, $\tau(t) / k(t)$ is constant. Thus, the rightmost term of the expression is constant as well. So, $\tau(t, t)$ grows at the same rate as $k(t)$, that is, $g$. Therefore, we can re-write the intertemporal government budget constraint as follows:

$$
\frac{\tau(t)}{k(t)}=\frac{-b \frac{\tau(t, t)}{k(t)}+r \tau_{k}}{r-g}
$$

(i) Considering $c(v, t)=\Delta^{-1}[a(v, t)+\tau(v, t)+h(v, t)]$ we calculate the growth rate of average consumption in the same way as above:

$$
g_{c}(t)=g_{c(v, .)}(t)+\left[\lambda-(b+\lambda) \frac{1+\frac{\tau(t)}{k(t)}-\beta \frac{\tau(t, t)}{k(t)}}{1+\frac{\tau(t)}{k(t)}+\frac{h(t)}{k(t)}}\right],
$$

where the term in square brackets represents the generation replacement effect, $\Gamma(t)$, in the presence of lump sum taxes and transfers. Clearly, $\Gamma$ declines in $\tau(t) / k(t)$, and it rises in $\tau(t, t) / k(t)$. The optimal allocation requires $c(v, t)=c(t), v \leq t \Leftrightarrow \Gamma=0$. It follows:

$$
\frac{\tau(t)}{k(t)}-\frac{\tau(t, t)}{k(t)}=-\frac{\lambda}{b}\left[\frac{b}{\lambda}-\frac{h(t)}{k(t)}\right] \Rightarrow \frac{\tau(t)}{k(t)}=\frac{-\lambda\left[\frac{b}{\lambda}-\frac{h(t)}{k(t)}\right]+r \tau_{k}}{r+b-g},
$$

where the second equality follows from (31). As discussed in Lemma $1(\mathrm{ii}), \operatorname{sgn}\{-[b / \lambda-$ $h / k]\}=\operatorname{sgn} \Gamma$. Thus, the first equality in (33) implies: $\operatorname{sgn}[\tau(t)-\tau(t, t)]=\left.\operatorname{sgn} \Gamma(t)\right|_{\tau(t, t)=\tau(t)=0}$. 
(ii) If $\left.\Gamma(t)\right|_{\tau(t, t)=\tau(t)=0}<0$, both terms in the numerator of the right hand side equation (33) are negative. Thus: $\tau(t)<0$. Next, consider $\left.\Gamma(t)\right|_{\tau(t, t)=\tau(t)=0}>0$. The optimal lump sum transfer system must lower $\Gamma$. Equation (31) shows that $\tau(t, t)$ and $\tau(t)$ cannot simultaneously be positive as $\tau(t, t)>0 \Rightarrow \tau(t)<0$. Also, $\tau(t, t)>0$ and $\tau(t)<0$ can be excluded, as this would unambiguously raise $\Gamma$ further. Thus, the optimal lump sum transfer system requires either $\tau(t, t)<0$ and $\tau(t)>0$ or $\tau(t, t)<0$ and $\tau(t)<0$. In both cases, $\tau(t, t)<0$.

Finally, define:

$\tau_{k}^{m} \equiv-\frac{r-g}{r}\left[1-\frac{\lambda(1-\alpha) A}{b(r+b+\lambda-g)}\right], \quad \tau_{k}^{m^{\prime}} \equiv \frac{b}{r} \frac{b(r+b-g-1)}{r-g}\left[1-\frac{\lambda(1-\alpha) A}{b(r+b+\lambda-g)}\right]$.

Considering (31) and (32), we can characterize the optimal lump sum transfer program as follows. Suppose $\left.\Gamma(t)\right|_{\tau(t, t)=\tau(t)=0}<0$, then $\tau(t)<0$, and $\tau(t, t) \gtrless 0 \Leftrightarrow \tau_{k} \gtrless \tau_{k}^{m}<0$. Suppose $\left.\Gamma(t)\right|_{\tau(t, t)=\tau(t)=0}>0$, then $\tau(t, t)<0$, and $\tau(t) \gtrless 0 \Leftrightarrow \tau_{k} \gtrless \tau_{k}^{m^{\prime}}<0$. $\|$

Proposition 5. According to (33), $\left[\frac{\tau(t)}{k(t)}-\frac{\tau(t, t)}{k(t)}\right]=-\frac{\lambda}{b}\left[\frac{b}{\lambda}-\frac{h(t)}{k(t)}\right]$. As $[h(t) / k(t)]=$ $[(1-\alpha) A] /[r+d+\lambda-g]$, the ratio $[h(t) / k(t)]$ rises in $g$. Given $\Gamma<0$, Proposition 1 implies that $(\partial g) /(\partial \eta)<0$. Consequently, $\left[\frac{\tau(t)}{k(t)}-\frac{\tau(t, t)}{k(t)}\right]$ declines in $\eta$ (that is, the expression becomes more negative). $\|$

Proposition 6. Consider (33). As $\Gamma>0,\left[\frac{\tau(t)}{k(t)}-\frac{\tau(t, t)}{k(t)}\right]>0$. As $[h(t) / k(t)]$ rises in $\eta$ $\left(\right.$ via $g$ ), so does $\left[\frac{\tau(t)}{k(t)}-\frac{\tau(t, t)}{k(t)}\right] . \|$

\section{References}

[1] Alpizar, F., Carlsson, F., and O. Johansson-Stenman (2005), How Much Do We Care About Absolute versus Relative Income and Consumption?, Journal of Economic Behavior \& Organization 56, 405-421. 
[2] Alvarez-Cuadrado, F., G. Monteiro, and S.J. Turnovsky (2004), Habit Formation, Catching Up with the Joneses, and Economic Growth, Journal of Economic Growth $9,47-80$.

[3] Blanchard, O. (1985), Debt, Deficits, and Finite Horizons, Journal of Political Economy $93,223-247$.

[4] Brekke, K.A., and R.B. Howarth (2002), Status, Growth and the Environment. Goods as Symbols in Applied Welfare Economics, Edward Elgar: Cheltenham.

[5] Calvo, G.A., and M. Obstfeld (1988), Optimal Time-Consistent Fiscal Policy with Finite Lifetimes, Econometrica 56, 411-432.

[6] Carlsson, F., O. Johansson-Stenman, and P. Martinsson (2003), Do You Enjoy Having More than Others? Survey Evidence of Positional Goods, Economica 74, 586-598.

[7] Carroll, C.D., J. Overland, and D.N. Weil (1997), Comparison Utility in a Growth Model, Journal of Economic Growth 2, 339-367.

[8] Corneo, G., and O. Jeanne (1997), On Relative Wealth Effects and the Optimality of Growth, Economics Letters 54, 87 - 92.

[9] Dupor, B., and W.F. Liu (2003), Jealousy and Equilibrium Overconsumption, American Economic Review 93, 423-428.

[10] Ferrer-i-Carbonell, A. (2005), Income and Well-Being: An Empirical Analysis of the Comparison Income Effect, Journal of Public Economics 89, 997-1019.

[11] Fisher, W.H., and B.J. Heijdra (2009), Keeping up with the Ageing Joneses, Journal of Economic Dynamics and Control 33, 53-64.

[12] Groth, C., and S. Smulders (2008), Robustness Problems in Endogenous Growth Theory, Working Paper, Economics Department: University of Copenhagen, Denmark. 
[13] Johansson-Stenman, O., F. Carlsson, and D. Daruvala (2002), Measuring Future Grandparents' Preferences for Equality and Relative Standing, Economic Journal $112,362-383$.

[14] Johansson-Stenman, O., and P. Martinsson (2006), Honestly, Why are You Driving a BMW? Journal of Economic Behavior \& Organization 60, 129-146.

[15] Liu, W.F., and S.J. Turnovsky (2005), Consumption Externalities, Production Externalities, and Long-Run Macroeconomic Efficiency, Journal of Public Economics 89, 1097-1129.

[16] Luttmer, E.F.P. (2005), Neighbors as Negatives: Relative Earnings and Well-Being, Quarterly Journal of Economics 120, 963-1002.

[17] McBride, M. (2001), Relative-Income Effects on Subjective Well-Being in the CrossSection, Journal of Economic Behavior 63 Organization 45, 251-278.

[18] Neumark, D., and A. Postlewaite (1998), Relative Income Concerns and the Rise in Married Women's Employment, Journal of Public Economics 70, 157-183.

[19] Rauscher, M. (1997), Conspicuous Consumption, Economic Growth, and Taxation, Journal of Economics 66, 35-42.

[20] Solnick, S.J., and D. Hemenway (1998), Is more always better? A Survey on Positional Concerns, Journal of Economic Behavior \& Organization 37, 373-383.

[21] Solnick, S.J., and D. Hemenway (2005), Are Positional Concerns Stronger in Some Domains than in Others?, American Economic Review 95, 147-151.

[22] Turnovsky, S.J., and G. Monteiro (2007), Consumption Externalities, Production Externalities, and Efficient Capital Accumulation under Time Non-separable Preferences, European Economic Review 51, 479-504. 
[23] Wendner, R., and L.H. Goulder (2008), Status Effects, the Excess Burden, and Optimal Levels of Public Goods Provision, Journal of Public Economics, 92, 1968 1985.

[24] Wendner, R. (2009), Conspicuous Consumption and Overlapping Generations, Research Memorandum 0901, Economics Department, University of Graz, Austria.

[25] Willenbockel, D. (2008), Social Time Preference Revisited, Journal of Population Economics 21, 609-622. 